\title{
Cólico del lactante: sobre lo que suele ser y lo que puede ser
}

\author{
A. García Salido ${ }^{a}$, A. Pérez Villena ${ }^{b}$ E. Pérez Suárez ${ }^{\mathrm{a}}$, MJ. Pérez García ${ }^{\mathrm{a}}$ \\ ${ }^{\circ}$ MIR-Pediatría. Unidad de Cuidados Intensivos Pediátricos. Hospital Universitario Niño Jesús. Madrid. España. \\ ${ }^{b}$ MIR-Pediatría. Unidad de Urgencias Pediátricas. Hospital Universitario Niño Jesús. Madrid. España.
}

\begin{abstract}
Resumen
Presentamos un paciente con clínica compatible con cólico del lactante, motivada por la presencia de vólvulo y malrotación intestinal, que asoció, además, ascitis quilosa. Describimos el ejercicio diagnóstico y el posterior tratamiento de este cuadro clínico realizando, a su vez, una breve revisión de la bibliografía disponible sobre ascitis quilosa de etiología no quirúrgica.
\end{abstract}

Palabras clave: Malrotación. Vólvulo. Ascitis. Cólico. Llanto.

Infant colic: what it usually is and what it can be

Abstract

We present a case of infant colic in relation to volvulus and intestinal malrotation associated with chylous ascites. The experience in the diagnosis and management of this condition is related with a review of the literature on chilous ascites in an infant without previous surgery.

Key words: Malrotation. Volvulus. Ascites. Colic. Crying.

\section{Introducción}

La clínica compatible con un cólico del lactante representa un motivo de consulta de elevada prevalencia en el servicio de urgencias pediátricas. Debido a que el paciente perteneciente a este grupo poblacional muestra una gran inespecificidad clínica, el cortejo de signos y síntomas de este cuadro puede resultar común a un gran número de entidades, lo que dificulta tanto su interpretación como el posterior manejo clínico".

Se describe un caso de cólico del lactante causado por la presencia de vólvulo y malrotación intestinal que se acompañó, tanto en el momento agudo como tras la cirugía correctora, de ascitis quilosa.

Alberto García Salido, citopensis@yahoo.es

Los autores declaran no presentar conflictos de intereses en relación con la preparación y publicación de este artículo. 


\section{Caso clínico}

Lactante varón de 27 días de edad que visitó el servicio de urgencias por presentar irritabilidad, llanto y rechazo parcial de la alimentación (realizada desde el nacimiento con lactancia artificial) durante 24 horas. No presentaba vómitos, alteración en las características y número de las deposiciones, fiebre ni decaimiento. Se refirieron cuadros previos de similares características y menor duración. Gestación gemelar con parto y período neonatal inmediato sin incidencias. No se refirieron antecedentes familiares de interés. Durante la exploración clínica se objetivó una gran distensión abdominal y llanto mode- rado en la palpación. No presentaba otros signos de interés y sus constantes tanto hemodinámicas como respiratorias se encontraban dentro de la normalidad para su edad. Ante la sospecha de un probable cólico del lactante se decidió realizar sondaje rectal para la evacuación del contenido gaseoso intestinal. Posteriormente, y debido a que el paciente se mostró confortado tras esta maniobra, se mantuvo en observación y se realizó una toma de lactancia artificial. Tras ésta, transcurridos pocos minutos, el paciente mostró de nuevo llanto, irritabilidad y distensión abdominal semejantes a los que habían motivado la consulta. En este momento, y

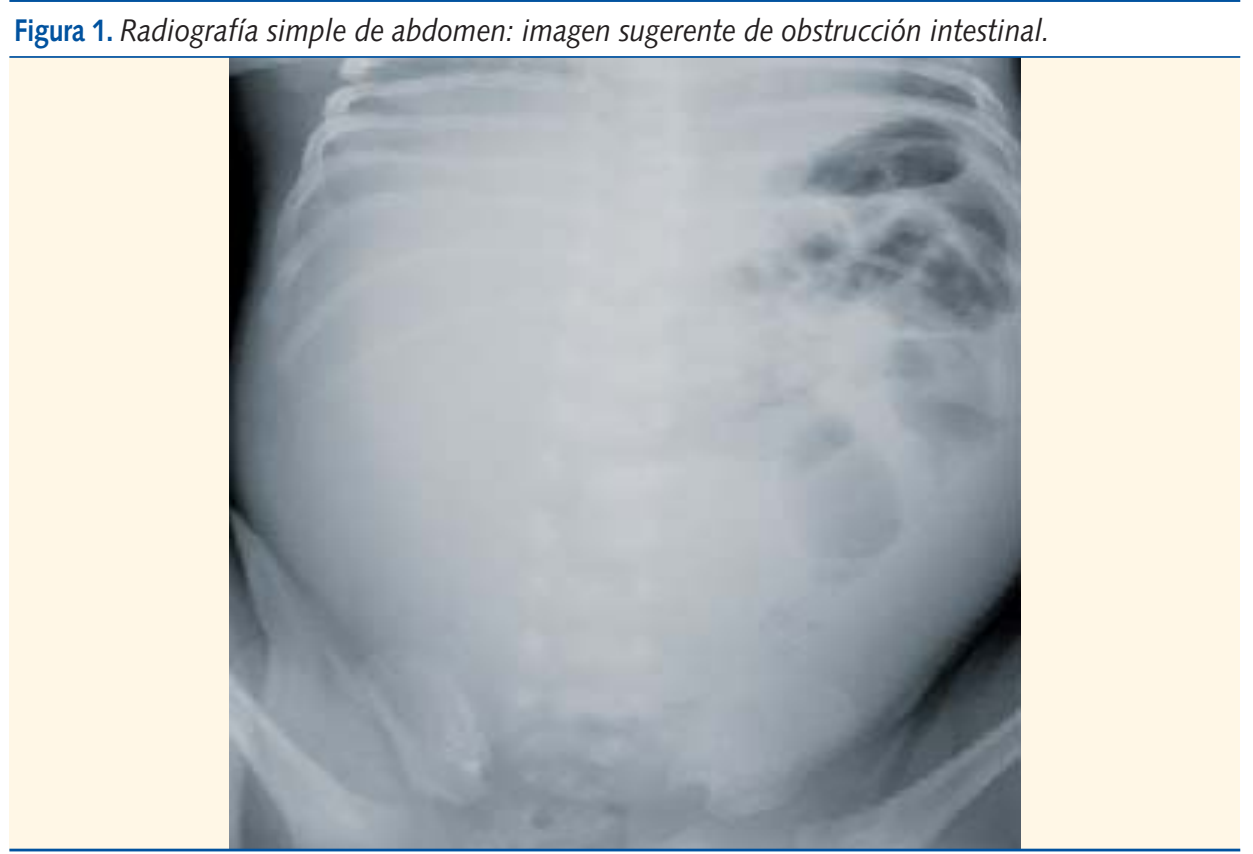


tras la reexploración física, se solicitó una radiografía abdominal (figura 1 ), en la cual se visualizó una imagen sugerente de obstrucción intestinal. Se completó el estudio de imagen mediante una ecografía abdominal; en ella se observó abundante líquido libre intraabdominal asociado a probable vólvulo o malrotación intestinal. Debido a los hallazgos descritos y ante la clínica del paciente, se decidió realizar abordaje quirúrgico.

Durante la intervención se obtuvo abundante contenido líquido blanquecino en la cavidad peritoneal -líquido compatible con quilo, tras realizar un estudio citológico, que presentaba abundantes trigli- céridos $(1.643 \mathrm{mg} / \mathrm{dl})$, con quilomicrones visibles mediante tinción de Negro-Sudán-y se objetivó la presencia de malrotación y vólvulo intestinal sin perforación intestinal asociada. Se realizó desvolvulación y fijación colónica mediante cirugía de Ladd. Tras la cirugía el paciente es enviado al servicio de cuidados intensivos pediátricos para observación y control clínico portando tubo de drenaje peritoneal y con profilaxis antibiótica.

El paciente permaneció a dieta absoluta durante 72 horas mostrando al reiniciar alimentación oral mala tolerancia y vómitos. Ante esto se inició nutrición parenteral que se mantuvo durante 12 días introduciendo

Figura 2. Enema opaco: dolicosigma con pequeño descenso del ángulo hepático del colon y ciego ligeramente desplazado hacia la línea media. 
paulatinamente ingesta enteral, desde el séptimo día postquirúrgico, mediante fórmula de alimentación rica en ácidos grasos de cadena media. Durante el ingreso mostró disminución progresiva de emisión de quilo a través del drenaje peritoneal por lo que, tras ecografía abdominal en la cual no se visualizó líquido libre, se retiró el sexto día tras la cirugía. Con objeto de visualizar la anatomía del intestino grueso se solicitó enema opaco (figura 2) en el que se vio dolicosigma con pequeño descenso del ángulo hepático del colon y ciego ligeramente desplazado hacia la línea media. Además se llevó a cabo ecocardiograma junto ecografía de aparato urinario descartándose patología concomitante en estas estructuras. Desde el punto de vista infeccioso el paciente recibió tratamiento antibiótico con vancomicina, meropenem y gentamicina mostrándose afebril y sin asociar signos o síntomas de infección durante todo el ingreso.

Finalmente recibió el alta con el diagnóstico de ascitis quilosa en relación con vólvulo y malrotación intestinal sin presentar nuevos episodios de volvulación.

\section{Discusión}

El llanto en el lactante es motivado con gran frecuencia por causas fisiológicas. En casos como el descrito, debido tanto a la ausencia de antecedentes clínicos de inte- rés como a su asociación con la distensión abdominal, se consideró como causa probable un cólico del lactante. Este diagnóstico, cuya prevalencia alcanza hasta un $20 \%{ }^{1}$ en lactantes sanos con edades comprendidas entre las 2 semanas y los 4 meses de vida, se debe establecer tras una correcta anamnesis y una exploración clínica que permita excluir otras posibles etiologías. No se conoce una etiología definida para el cólico del lactante, de la misma manera que no existe un tratamiento determinado. En el caso que nos ocupa se observa cómo, tras considerar como causa del llanto la entidad anteriormente descrita, el paciente no mostró mejoría tras la liberación de contenido gaseoso intestinal. Así, se sospechó la existencia de otra causa en el origen del llanto, la irritabilidad y la distensión del abdomen y se solicitaron de este modo pruebas complementarias de imagen que permitieron definir una sospecha y plantear un tratamiento, en este caso quirúrgico, para ella.

En cuanto a la presencia de quilo en la cavidad abdominal ésta constituye una entidad de baja prevalencia en la población infantil no sometida a intervención quirúrgica. Se relaciona con alteraciones congénitas del drenaje linfático asociadas a malformaciones (54\%), obstrucción (27\%, como el caso que nos ocupa $\left.{ }^{2}\right)$ o traumatismo $(19 \% \text { maltrato infantil) })^{3}$. A 
su vez supone una entidad bien conocida derivada generalmente de intervenciones quirúrgicas (generalmente torácicas o retroperitoneales) o infiltración neoplásica del sistema linfático intraabdominal ${ }^{4}$. La presencia de líquido linfático en la cavidad abdominal se puede producir mediante exudación (típica de infiltración maligna), liberación directa tras fístula linfática o debido a fenómenos obstructivos o malformativos que permitan el desarrollo de grandes vasos linfáticos con facilidad para liberar su contenido ${ }^{4}$.

Desde el punto de vista clínico la ascitis quilosa se acompaña de signos y síntomas clínicos muy inespecíficos apareciendo la distensión abdominal hasta en un $80 \%$ de los casos $^{3}$.

Esta patología implica tanto la pérdida de nutrientes contenidos en la linfa como un compromiso inmunológico, comúnmente objetivado cómo linfocitopenia, derivado del abundante contenido en linfocitos por parte de ésta ${ }^{4}$. El tratamiento no debe demorarse en consecuencia, pues asocia una mortalidad de hasta el $24 \%{ }^{4}$ en algunas series, dirigiéndose por un lado a prevenir la sobreinfección y por otro a evitar la desnutrición.

En pacientes en los que la causa de la ascitis quilosa no está en relación con intervención quirúrgica previa se recomienda la observación clínica junto con sopor- te farmacológico como opción terapéuti$\mathrm{ca}^{5}$ debido a la alta tasa de curación obtenida con esta opción terapeútica (hasta un $87,25 \%)^{6}$.

Desde el punto de vista nutricional se recomienda el reinicio de la alimentación enteral mediante fórmula rica en ácidos grasos de cadena media ${ }^{7}$ siendo necesario mantener a dieta absoluta al paciente hasta la desaparición o disminución franca del débito linfático. En caso de persistencia del mismo es común el uso de análogos de la somatostatina como el octeótride.

El interés de este caso radica en que si bien es importante conocer la existencia y el tratamiento de enfermedades de baja incidencia en el paciente pediátrico, como es la ascitis quilosa sin antecedentes de intervención quirúrgica, es aún más relevante recalcar la necesidad de una adecuada aproximación diagnóstica a todo lactante con sospecha de cólico abdominal. De algún modo no debemos olvidar que aunque la medicina es el arte de lo frecuente puede que sea lo infrecuente a lo que nos enfrentemos en la consulta.

\section{Agradecimientos}

A los servicios de Urgencias, Cuidados Intensivos Pediátricos y Cirugía Pediátrica del Hospital Infantil Universitario Niño Jesús de Madrid. 


\section{Bibliografía}

1. Sanjosé González MA, Tabernero Carrascosa M. Llanto. Cólico del lactante. En: Ruiz Domínguez JA, Montero Reguera R, Hernández González N, editores. Manual de Diagnóstico y Terapéutica en Pediatría. 4. ${ }^{a}$ ed. Madrid: Publimed Editores; 2003. p. 33-7.

2. Chye JK, Lim CT, Van der Heuvel M. Neonatal chylous ascites: report of three cases and review of the literature. Pediatr Surg Int. 1997;12:296-8.

3. Cochran WJ, Klish WJ, Brown MR, Lyons JM, Curtis T. Chylous ascites in infants and children: a case report and literature review. J Pediatr Gastroenterol Nutr. 1985;4:668-73.
4. Aalami OO, Allen DB, Organ CH. Chylous ascites: A collective review. Surgery. 2000;128:7618.

5. Unger SW, Chandler JG. Chylous ascites in infants and children. Surgery. 1983;93:455-61.

6. Densupsoontorn NS, Jirapinyo $P$, Wongarn $R$, Thamonsiri N, Nana A, Laohaprasitiporn D, et al. Management of chylothorax and chylopericardium in pediatric patients: experiences at Siriraj Hospital, Bangkok. Asia Pac J Clin Nutr. 2005;14: 182-7.

7. Jernite $M$, Donato L, Favre R, Haddad J, Esposito $M$, Messer J. Medical treatment of chylous effusions in newborn infants. Apropos of 3 cases. Arch Fr Pediatr. 1992;49:811-4. 